\title{
Early post-stress LV dyssynchrony: A new marker for significant CAD
}

\author{
Wael AlJaroudi, MD, FACC, FASNC ${ }^{a}$ \\ a Division of Cardiovascular Disease, American University of Beirut Medical Center, Beirut, \\ Lebanon
}

doi: $10.1007 / \mathrm{s} 12350-014-9973-6$

\section{See related article, pp. 1048-1056}

Single photon emission computed tomography (SPECT) myocardial perfusion imaging (MPI) remains one of the most commonly ordered tests for the detection of ischemia and significant coronary artery disease (CAD). ${ }^{1}$ A coronary artery territory perfused by a significantly narrowed vessel has limited coronary flow reserve; hence, during peak stress, there are less blood flow and tracer uptake in that region as compared to normal myocardial. The detection of reversible perfusion defect, therefore, depends on the relative flow between myocardial territories. ${ }^{2}$ In patients with multivessel disease, the coronary flow reserve is reduced in all territories leading to what has been described as balanced ischemia phenomenon. ${ }^{3}$ The latter poses a challenge as patients with multi-vessel CAD may have "normal" MPI and could be miss-diagnosed or more commonly assumed to have less extensive CAD. ${ }^{3}$ However, other complementary tools have helped recognize the presence of high ischemic burden even when the perfusion images say otherwise. Among them, transient ischemic dilation (TID), ${ }^{4}$ increased lung uptake of Tl-201, ${ }^{5}$ and left ventricular (LV) stunning (i.e., decrease in early post-stress LV ejection fraction [EF]). These tools, nevertheless, have limitations: the diagnostic accuracy of TID is low; Tl-201 is rarely used and has been replaced by Tc-99m; and LV stunning is often missed or underestimated since imaging with SPECT

Reprint requests: Wael AlJaroudi, MD, FACC, FASNC, Division of Cardiovascular Disease, American University of Beirut Medical Center, Beirut, Lebanon; wa53@aub.edu.lb

Funding None.

J Nucl Cardiol 2014;21:1057-61.

$1071-3581 / \$ 34.00$

Copyright (C) 2014 American Society of Nuclear Cardiology. does not occur at peak stress giving enough time for LV recovery. ${ }^{6}$

LV mechanical dyssynchrony indices have been proposed as additional tools to help detect myocardial stunning and ischemia-induced dyssynchronous contraction. The technique was developed by $\mathrm{Chen}^{7}$ almost a decade ago. It calculates a phase value for each of sampled regions $(>600)$ that represent the onset of mechanical contraction. The standard deviation (phase $\mathrm{SD}$ ) and 95\% bandwidth (histogram bandwidth) represent the LV dyssynchrony indices: the bigger the numbers, the greater the dyssychrony. These indices are derived retrospectively from prior acquired and saved images, with no additional radiation burden or cost. They are automated, reproducible with small inter and intra-observer variability, ${ }^{8}$ repeatable, ${ }^{9}$ and have been validated against two ${ }^{10}$ and three-dimensional echocardiography. ${ }^{11}$ The initial application of the technique was in identifying the optimal patients with cardiomyopathy who would derive most benefit from cardiac resynchronization therapy. ${ }^{12}$ Since then, the technique proved not only to be a valuable tool in detecting mechanical dyssynchrony, but also providing prognostic information and risk stratifying patients with various co-morbidities (ischemic, ${ }^{13}$ non-ischemic cardiomyopathy, ${ }_{14}^{14}$ implantable defibrillators, ${ }^{15}$ end-stage renal disease ${ }^{16,17}$ ). More recently, the technique has been used to detect ischemiainduced myocardial dyssynchrony and multi-vessel $\mathrm{CAD}$ by comparing stress and rest indices ${ }^{18-20}$ (Table 1).

The concept of stress-induced changes in LV dyssynchrony is not new. In patients with normal MPI undergoing gated positron emission tomography (PET), LV mechanical dyssynchrony indices were smaller when derived from peak stress vs rest gated images, irrespective of the resting LVEF. ${ }^{21}$ These findings were thought to be related to stress-induced increased hyperemia leading to more signal and less noise during phase analysis, ${ }^{22}$ but also to improved LV contractility that can occur with vasodilator testing. Similarly, Salimian et $\mathrm{al}^{23}$ showed that 
Table 1. Potential clinical applications of left ventricular mechanical dyssynchrony by phase analysis

Selection of optimal candidates for cardiac resynchronization therapy

Guiding placement of LV lead at the site of latest onset mechanical dyssynchrony

Detecting stress-induced global and regional worsening of LV dyssynchrony as a marker of myocardial stunning and significant ischemia

Differentiating ischemic vs non-ischemic cardiomyopathy based on the presence or absence of stress-induced worsening of LV dyssynchrony

Risk stratification and prognostication in patients with: Ischemic cardiomyopathy

Non-ischemic cardiomyopathy

Implantable cardiac defibrillators

End-stage renal disease

dobutamine stress amplifies LV synchronicity leading to lower dyssynchrony indices.

In patients with stress-induced ischemia, there is initially a supply-demand mismatch that translates into perfusion abnormality followed by abnormal myocardial relaxation, contraction, and LV stunning. Therefore, stress-induced worsening of LV mechanical dyssynchrony is potentially a reflection of myocardial stunning, and therefore a potential marker for multi-vessel CAD. However, optimal detection of stress-induced worsening of LV dyssynchrony is dependent on the time delay between peak stress and imaging. Late post-stress imaging (30-60 minutes), as is the case with Tc-99m SPECT imaging, often results in recovery of LV stunning with no residual detectable change in LV dyssynchrony (stress vs rest) at the time of imaging, even in the presence of large ischemic burden. ${ }^{24,25}$ On the other hand, early post-stress imaging that is more feasible with $\mathrm{Tl}$ 201 (5-10 minutes) captures stress-induced changes in LV dyssynchrony. ${ }^{26}$ Indeed, dyssynchronous contraction in the ischemic region during peak stress, leads to deterioration of LV synchrony as opposed to the normal myocardium that retains its synchrony. ${ }^{26}$ As a further validation of their initial findings, Huang et $\mathrm{al}^{18}$ showed that early post-stress LV mechanical dyssynchrony by phase analysis of gated Tl-201 SPECT MPI is a useful tool to detect multi-vessel CAD.

In the current study, 144 consecutive patients who underwent stress/rest Tl-201 SPECT MPI and

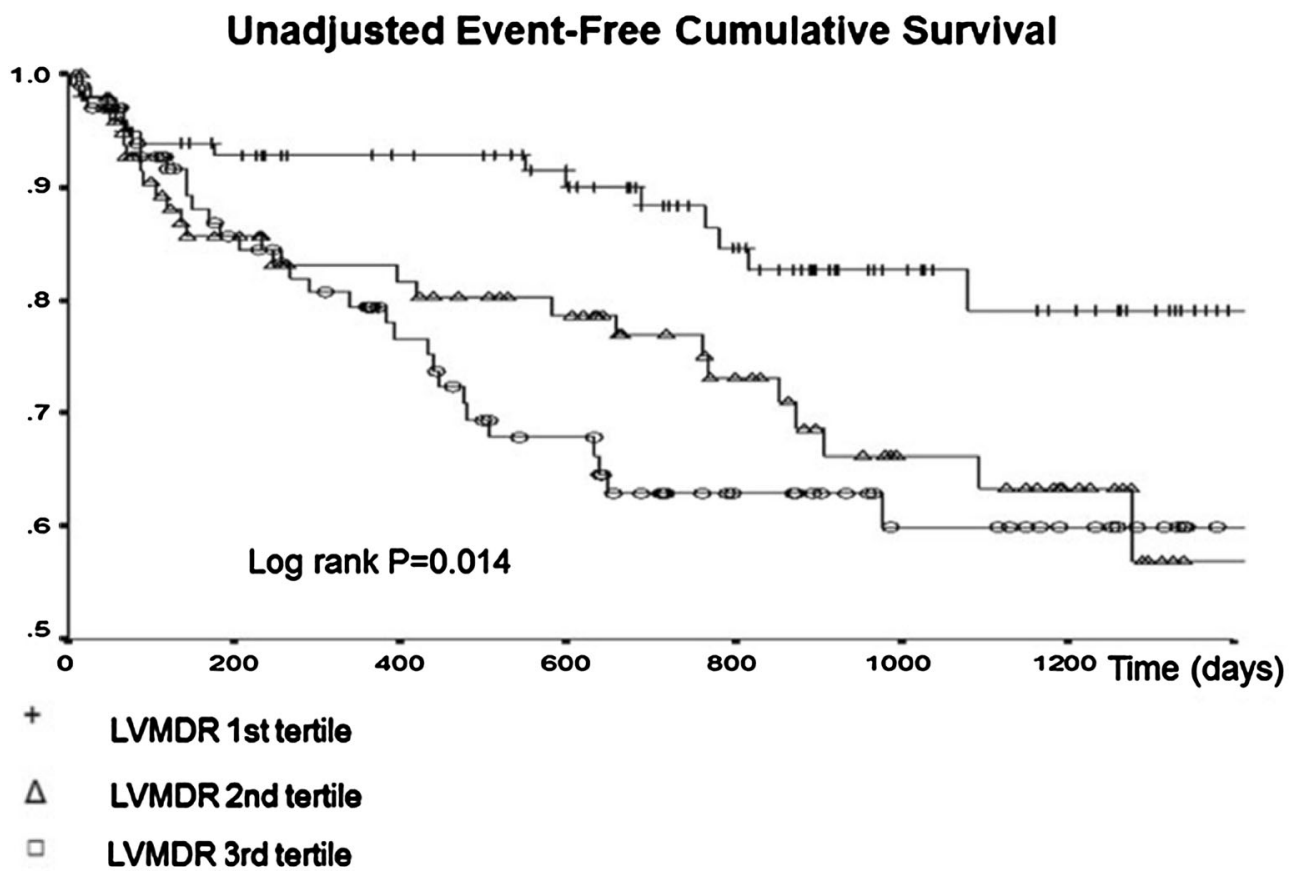

Figure 1. Unadjusted Kaplan-Meier survival curves illustrating survival of patients with ischemic cardiomyopathy undergoing stress/rest Rb82 PET, stratified by tertiles of change in left ventricular mechanical dyssynchrony index reserve (LVMDR = stress-rest phase SD). There was significant increase in all-cause mortality among patients with the most worsening of stress phase SD (15\% vs $26 \%$ vs $31 \%$ for the 1 st, 2 nd, and 3 rd tertiles, respectively, $\log$ rank $P=0.014$ ). (Reproduced with permission from AlJaroudi et al. J Nucl Cardiol 2012;19:958-969 [Figure 4] ${ }^{20}$ ). Positron emission tomography (PET). 

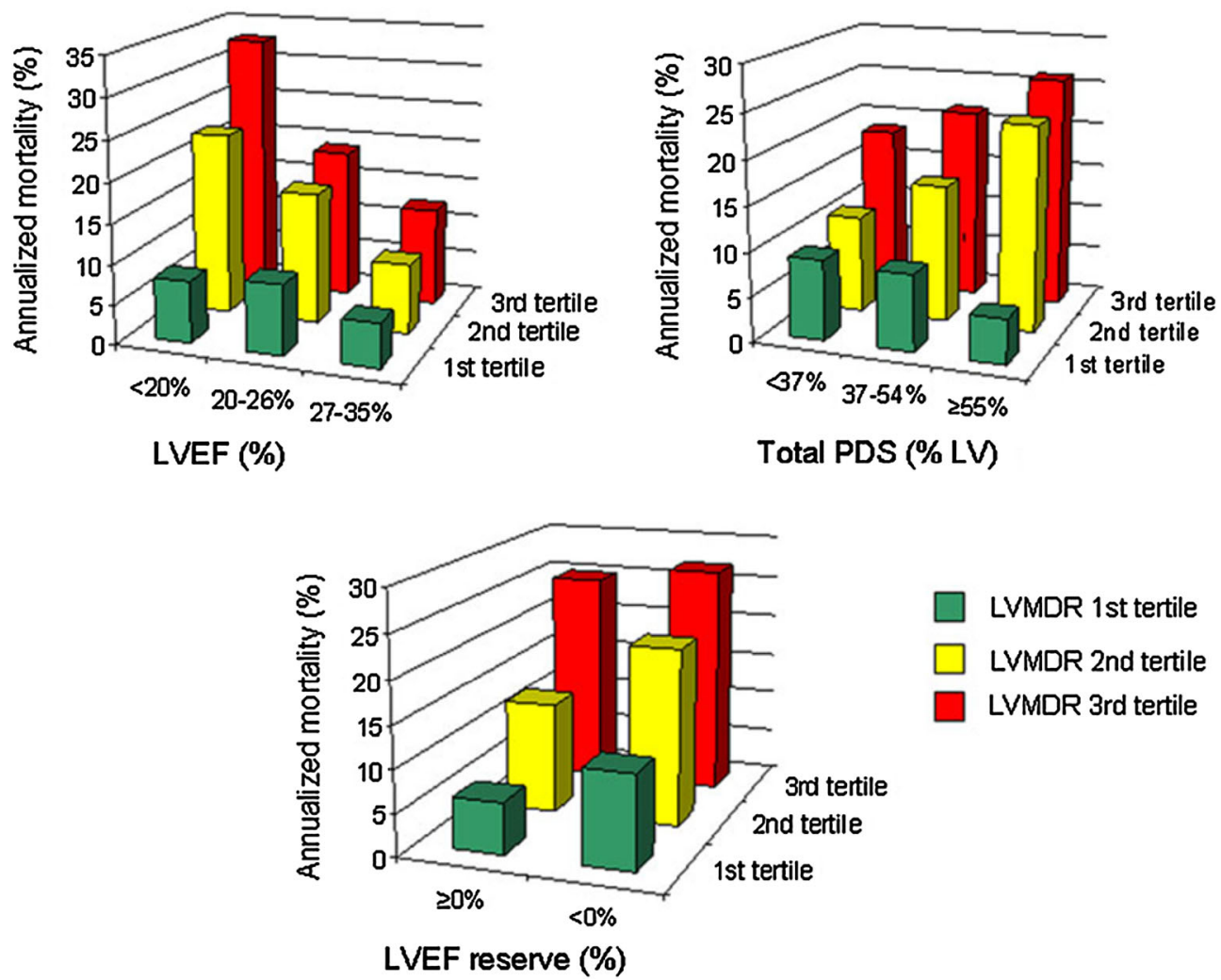

LVMDR 1st tertile

LVMDR 2nd tertile

LVMDR 3rd tertile

Figure 2. Three-dimensional bar diagram plot illustrating incremental increase in mortality among patients with more significant stress-induced worsening of LV dyssynchrony (stratified by tertiles) and across different ranges of LVEF, perfusion defect size and LVEF reserve (Reproduced with permission from AlJaroudi et al, J Nucl Cardiol 2012;19:958-969 [Figure 5] ${ }^{20}$ ). LVMDR = stressrest phase SD.

subsequent coronary angiography were evaluated. ${ }^{18}$ There were 57 patients with no CAD, 32 with 1-vessel disease, 36 with 2-vessel disease, and 19 with 3-vessel disease. Patients with multi-vessel CAD had significantly more global and regional LV dyssynchrony at early post-stress imaging as compared to rest. Also, dysysnchrony assessment correctly classified more patients with multi-vessel disease as compared to perfusion abnormalities and $\mathrm{EF}$ changes alone. ${ }^{18}$ Using similar concept, Igarashi et al ${ }^{19}$ showed that patients with CAD had significantly higher global changes of phase SD with stress, and were able to differentiate patients with heart failure as ischemic vs non-ischemic etiologies.

The ability to detect and quantify regional LV mechanical dyssynchrony is obviously a leap forward in the technique of phase analysis and has several advantages and clinical utilities (Table 1). Furthermore, the measurements of LV dyssynchrony indices were performed in a Core lab by experts who developed the technique, and in a blinded manner adding more clinical validity to the results. The study was well designed and the results are quite compelling and encouraging to pursue larger prospective validation studies.

There are some limitations to the study, however. First, the cut-off of phase SD $4.3^{\circ}$ as being a significant change in LV dyssynchrony $(z$ score of $1.65 \times$ the standard deviation of phase SD between two serial scans $2.6^{\circ}$ ]) was based on images obtained by Tc- $99 \mathrm{~m} .{ }^{27} \mathrm{In}$ the current study, Tl-201 was used which has lower energy, and results in more background noise and poorer image quality that may lead to higher variation in dyssynchrony indices on serial scans; however, the authors did use a prolonged acquisition protocol to increase count density of the images. Second, although the correlation between change in phase SD and ischemic burden was statistically significant, it was fair to modest at best $(r=0.25)$ with wide scatter resulting in reduced diagnostic accuracy. For instance, there were several patients with significant worsening of LV dyssynchrony yet with no or minimal ischemic burden. Third, there were no data available on the QRS duration and baseline EF that do affect LV dyssynchrony indices. Last, significant $\mathrm{CAD}$ based on the anatomic definition of 
epicardial stenosis $\geq 70 \%$, although widely accepted in the community, does not always translate into ischemia; conversely, ischemic myocardium can be detected with less epicardial stenosis depending on the microvascular bed. Hence, the use of quantitative flow data based on PET or fractional flow reserve in the catheterization laboratory would have been a more optimal way to define significant ischemia and correlate it with the MPI. ${ }^{28}$

While Tl-201 imaging allows early post-stress imaging and theoretically more detection of stressinduced LV dyssynchrony and multi-vessel disease, it is less commonly used these days. This is in part related to the lower quality images obtained, high background noise, and significantly more radiation burden as compared to Tc-99m. With the introduction of PET MPI, and more recently dynamic PET with coronary flow reserve assessment, the detection of multi-vessel CAD has become less challenging. ${ }^{29}$ PET MPI provides even better quality images, lower radiation dose, and the ability to obtained immediate peak-stress images. The latter can be used to identify true LV stunning and worsening of LV dyssynchrony. Our group has recently showed the clinical utility and prognostic value of LV mechanical dyssynchrony in patients with ischemic ${ }^{13}$ and non-ischemic ${ }^{14}$ cardiomyopathy undergoing gated PET. Moreover, worsening of LV dyssynchrony at peak stress was an independent predictor of mortality and provided incremental value to the traditional risk factors in a study of 489 patients with ischemic cardiomyopathy undergoing rest/stress Rb-82 gated PET $^{20}$ (Figures 1, 2). PET imaging, however, is not widely available, and is significantly more expensive than SPECT imaging.

LV mechanical dyssynchrony by phase analysis has proved to be a unique tool that provides relevant diagnostic and prognostic data. The detection of multi-vessel disease using stress vs rest changes is yet another potential application for the technique. It is time to start reporting it regularly on all clinical studies, have it integrated in the reports, and build a large multicenter database.

\section{Conflict of interest}

None.

\section{References}

1. Zoghbi GJ, Iskandrian AE. Nuclear cardiac imaging. Exercise myocardial perfusion imaging (chapter 15). 4th ed. Oxford: Oxford University Press; 2008.

2. Naya M, Murthy VL, Taqueti VR, Foster CR, Klein J, Garber M, et al. Preserved coronary flow reserve effectively excludes high- risk coronary artery disease on angiography. J Nucl Med 2014; 55:248-55.

3. Berman DS, Kang X, Slomka PJ, Gerlach J, de Yang L, Hayes $\mathrm{SW}$, et al. Underestimation of extent of ischemia by gated SPECT myocardial perfusion imaging in patients with left main coronary artery disease. J Nucl Cardiol 2007;14:521-8.

4. Emmett L, Ng A, Ha L, Russo R, Mansberg R, Zhao W, et al. Comparative assessment of rest and post-stress left ventricular volumes and left ventricular ejection fraction on gated myocardial perfusion imaging (MPI) and echocardiography in patients with transient ischaemic dilation on adenosine MPI: Myocardial stunning or subendocardial hypoperfusion? J Nucl Cardiol 2012; 19:735-42.

5. Daou D, Coaguila C, Delahaye N, Houzet F, Lebtahi R, Le Guludec D. Discordance between exercise SPECT lung Tl-201 uptake and left ventricular transient ischemic dilation in patients with CAD. J Nucl Cardiol 2004;11:53-61.

6. Johnson LL, Verdesca SA, Aude WY, Xavier RC, Nott LT, Campanella MW, et al. Postischemic stunning can affect left ventricular ejection fraction and regional wall motion on poststress gated sestamibi tomograms. J Am Coll Cardiol 1997; 30:1641-8.

7. Chen J, Garcia EV, Folks RD, Cooke CD, Faber TL, Tauxe EL, et al. Onset of left ventricular mechanical contraction as determined by phase analysis of ECG-gated myocardial perfusion SPECT imaging: Development of a diagnostic tool for assessment of cardiac mechanical dyssynchrony. J Nucl Cardiol 2005;12:687-95.

8. Trimble MA, Velazquez EJ, Adams GL, Honeycutt EF, Pagnanelli RA, Barnhart HX, et al. Repeatability and reproducibility of phase analysis of gated single-photon emission computed tomography myocardial perfusion imaging used to quantify cardiac dyssynchrony. Nucl Med Commun 2008;29:374-81.

9. Lin X, Xu H, Zhao X, Folks RD, Garcia EV, Soman P, et al. Repeatability of left ventricular dyssynchrony and function parameters in serial gated myocardial perfusion SPECT studies. J Nucl Cardiol 2010;17:811-6.

10. Henneman MM, Chen J, Ypenburg C, Dibbets P, Bleeker GB, Boersma E, et al. Phase analysis of gated myocardial perfusion single-photon emission computed tomography compared with tissue doppler imaging for the assessment of left ventricular dyssynchrony. J Am Coll Cardiol 2007;49:1708-14.

11. Marsan NA, Henneman MM, Chen J, Ypenburg C, Dibbets $P$, Ghio S, et al. Real-time three-dimensional echocardiography as a novel approach to quantify left ventricular dyssynchrony: A comparison study with phase analysis of gated myocardial perfusion single photon emission computed tomography. J Am Soc Echocardiogr 2008;21:801-7.

12. Henneman MM, Chen J, Dibbets-Schneider P, Stokkel MP, Bleeker GB, Ypenburg C, et al. Can LV dyssynchrony as assessed with phase analysis on gated myocardial perfusion SPECT predict response to CRT? J Nucl Med 2007;48:1104-11.

13. AlJaroudi W, Alraies MC, Hachamovitch R, Jaber WA, Brunken $\mathrm{R}$, Cerqueira MD, et al. Association of left ventricular mechanical dyssynchrony with survival benefit from revascularization: A study of gated positron emission tomography in patients with ischemic LV dysfunction and narrow QRS. Eur J Nucl Med Mol Imaging 2012;39:1581-91.

14. Goldberg AS, Alraies MC, Cerqueira MD, Jaber WA, Aljaroudi WA. Prognostic value of left ventricular mechanical dyssynchrony by phase analysis in patients with non-ischemic cardiomyopathy with ejection fraction $35-50 \%$ and QRS $<150 \mathrm{~ms}$. J Nucl Cardiol 2014;21:57-66.

15. Aljaroudi WA, Hage FG, Hermann D, Doppalapudi H, Venkataraman R, Heo J, et al. Relation of left-ventricular dyssynchrony 
by phase analysis of gated spect images and cardiovascular events in patients with implantable cardiac defibrillators. J Nucl Cardiol 2010;17:398-404.

16. Aggarwal H, AlJaroudi WA, Mehta S, Mannon R, Heo J, Iskandrian AE, et al. The prognostic value of left ventricular mechanical dyssynchrony using gated myocardial perfusion imaging in patients with end-stage renal disease. J Nucl Cardiol 2014;21:73946.

17. AlJaroudi W, Aggarwal H, Venkataraman R, Heo J, Iskandrian AE, Hage FG. Impact of left ventricular dyssynchrony by phase analysis on cardiovascular outcomes in patients with end-stage renal disease. J Nucl Cardiol 2010;17:1058-64.

18. Huang W, Huang C, Lee C, Chem C, Chuang-Uei H, Chen J. Relation of early post-stress left ventricular dyssynchrony and the extent of angiographic coronary artery disease. J Nucl Cardiol; 2014 (epub ahead of print).

19. Igarashi $Y$, Chikamori T, Hida S, Tanaka H, Shiba C, Usui $Y$, et al. Usefulness of phase analysis to differentiate ischemic and non-ischemic etiologies of left ventricular systolic dysfunction in patients with heart failure. Circ J 2013;78:141-50.

20. AlJaroudi W, Alraies MC, Menon V, Brunken RC, Cerqueira MD, Jaber WA. Predictors and incremental prognostic value of left ventricular mechanical dyssynchrony response during stress-gated positron emission tomography in patients with ischemic cardiomyopathy. J Nucl Cardiol 2012;19:958-69.

21. AlJaroudi W, Alraies MC, DiFilippo F, Brunken RC, Cerqueira MD, Jaber WA. Effect of stress testing on left ventricular mechanical synchrony by phase analysis of gated positron emission tomography in patients with normal myocardial perfusion. Eur J Nucl Med Mol Imaging 2012;39:665-72.

22. AlJaroudi W, Jaber WA, Cerqueira MD. Effect of tracer dose on left ventricular mechanical dyssynchrony indices by phase analysis of gated single photon emission computed tomography myocardial perfusion imaging. J Nucl Cardiol 2012;19:63-72.

23. Salimian S, Thibault B, Finnerty V, Gregoire J, Harel F. The effects of dobutamine stress on cardiac mechanical synchrony determined by phase analysis of gated SPECT myocardial perfusion imaging in a canine model. J Nucl Cardiol 2014;21:375-83.

24. Aljaroudi W, Koneru J, Heo J, Iskandrian AE. Impact of ischemia on left ventricular dyssynchrony by phase analysis of gated single photon emission computed tomography myocardial perfusion imaging. J Nucl Cardiol 2011;18:36-42.

25. Zhou Y, Li D, Feng J, Yuan D, Patel Z, Cao K, et al. Left ventricular dyssynchrony parameters measured by phase analysis of post-stress and resting gated spect myocardial perfusion imaging. World J Nucl Med 2013;12:3-7.

26. Chen CC, Shen TY, Chang MC, Hung GU, Chen WC, Kao CH, et al. Stress-induced myocardial ischemia is associated with early post-stress left ventricular mechanical dyssynchrony as assessed by phase analysis of $201 \mathrm{Tl}$ gated spect myocardial perfusion imaging. Eur J Nucl Med Mol Imaging 2012;39:1904-9.

27. Zhou Y, Faber TL, Patel Z, Folks RD, Cheung AA, Garcia EV, et al. An automatic alignment tool to improve repeatability of left ventricular function and dyssynchrony parameters in serial gated myocardial perfusion SPECT studies. Nucl Med Commun 2013;34:124-9.

28. Kakouros N, Rybicki FJ, Mitsouras D, Miller JM. Coronary pressure-derived fractional flow reserve in the assessment of coronary artery stenoses. Eur Radiol 2013;23:958-67.

29. Van Tosh A, Votaw JR, Reichek N, Palestro CJ, Nichols KJ. The relationship between ischemia-induced left ventricular dysfunction, coronary flow reserve, and coronary steal on regadenoson stress-gated $(82) \mathrm{Rb}$ pet myocardial perfusion imaging. J Nucl Cardiol 2013;20:1060-8. 